\title{
Effects of an 8-week yoga program on sustained attention and discrimination function in children with attention deficit hyperactivity disorder
}

\author{
Chien-Chih Chou ${ }^{1}$, Chung-Ju Huang ${ }^{\text {Corresp. } 1}$ \\ ${ }^{1}$ Graduate Institute of Sport Pedagogy, University of Taipei, Taipei, Taiwan \\ Corresponding Author: Chung-Ju Huang \\ Email address: crhwang_tpec@yahoo.com.tw
}

This study investigated whether a yoga exercise intervention influenced the sustained attention and discrimination function in children with ADHD. Forty-nine participants (mean age $=10.50$ years) were assigned to either a yoga exercise or a control group. Participants were given the Visual Pursuit Test and Determination Test prior to and after an eight-week exercise intervention (twice per week, 40 min per session) or a control intervention. Significant improvements in accuracy rate and reaction time of the two tests were observed over time in the exercise group compared with the control group. These findings suggest that alternative therapies such as yoga exercises can be complementary to behavioral interventions for children with attention and inhibition problems. Schools and parents of children with ADHD should consider alternatives for maximizing the opportunities that children with ADHD can engage in structured yoga exercises. 
1 Title: Effects of an 8-Week Yoga Program on Sustained Attention and Discrimination Function

2 in Children with Attention Deficit Hyperactivity Disorder

3 Running head: Yoga and Attention Performance in ADHD

\section{Authors: Chien-Chih Chou \& Chung-Ju Huang}

5 Affiliation: Graduate Institute of Sport Pedagogy, University of Taipei, Taiwan

\section{Chien-Chih Chou}

7 Graduate Institute of Sport Pedagogy, University of Taipei

8 Address: 101, Sec. 2, Zhongcheng Rd., Taipei 111, Taiwan, R.O.C.

9 E-mail: ccc4453@gmail.com

\section{Chung-Ju Huang (Corresponding author)}

11 Graduate Institute of Sport Pedagogy, University of Taipei

12 Address: 101, Sec. 2, Zhongcheng Rd., Taipei 111, Taiwan, R.O.C.

13 E-mail: crhwang_tpec@yahoo.com.tw

14 Authors Note:

15 Chung-Ju Huang and Chien-Chih Chou are professors with the Graduate Institute of Sport 16 Pedagogy, University of Taipei, Taiwan. 
19 Effects of an 8-Week Yoga Program on Sustained Attention and Discrimination Function in Children with Attention Deficit Hyperactivity Disorder

\section{Abstract}

23 attention and discrimination function in children with ADHD. Forty-nine participants (mean age

$24=10.50$ years) were assigned to either a yoga exercise or a control group. Participants were given

25 the Visual Pursuit Test and Determination Test prior to and after an eight-week exercise

26 intervention (twice per week, 40 min per session) or a control intervention. Significant

27 improvements in accuracy rate and reaction time of the two tests were observed over time in the

28 exercise group compared with the control group. These findings suggest that alternative therapies

29 such as yoga exercises can be complementary to behavioral interventions for children with

30 attention and inhibition problems. Schools and parents of children with ADHD should consider

31 alternatives for maximizing the opportunities that children with ADHD can engage in structured

32 yoga exercises. 
Effects of an 8-Week Yoga Program on Sustained Attention and Discrimination Function in Children with Attention Deficit Hyperactivity Disorder

\section{Introduction}

commonly occurs among school-aged children, with approximately 5-8 percent of children being

44 affected. Moreover, the symptoms of the disorder persist into adulthood in up to 60 percent of

45 childhood cases, meaning that roughly 4 percent of adults suffer from ADHD (Barbaresi et al.,

46 2002; Froehlich et al., 2007). The disorder is characterized by inappropriate attention,

47 impulsivity and/or hyperactivity which may cause a variety of problems including academic

48 difficulties, impaired social skills, and strained parent-child relationships (Harpin, 2005). In

49 regard to underlying mechanisms of ADHD, several models have been proposed such as

50 maturational lag, cortical hypo-arousal, and developmental deviation (Barry, Clarke, \&

51 Johnstone, 2003). Given that long-term medications can ameliorate ADHD symptoms but may

52 have negative side-effects such as sleep disturbances, reduced appetite, and mood disorders

53 (Pliszka, 2007), physical activity can serve as a low-risk treatment for ADHD symptoms. A

54 recent systematic review has suggested that physical activity shows promise as an effective 
55 treatment, reporting improvement in measures of interference control, set shifting, consistency in

56 response speed, vigilance and impulsive control among individuals with ADHD (Halperin,

57 Berwid, \& O’Neill, 2014). Therefore, further examining beneficial effects of different forms of

58 physical activity on attention performance in children with ADHD can extend the current

59 understanding of the association between physical activity and cognition function of individuals

60 with ADHD.

61 Yoga exercise has been found to be a feasible school intervention for children with

62 emotional and behavioural disorders and can be effective in ameliorating the symptoms that also

63 pervasively occur in children with ADHD, such as inattention and bad adaptive skills in class

64 (Steiner, Sidhu, Pop, Frenette, \& Perrin, 2013). Different from normal physical exercise, yoga

65 practicing steers individuals to master certain breathing techniques, postures, and cognitive

66 control which can help promote self-control, attention, body awareness, and stress management

67 (Kimbrough, Balkin, \& Rancich, 2007). Previous studies have demonstrated that yoga shows

68 promise as an intervention for a variety of social, emotional, behavioral, and cognitive ailments

69 (Diamond \& Lee, 2011). Although previous research regarding the effects of yoga on ADHD

70 symptoms is somewhat limited, several studies have been conducted. Some studies employing

71 yoga as a treatment for ADHD reported beneficial effects such as reduced hyperactivity,

72 inattention, and anxiety, and improved peer relationship quality and sleep patterns (Harrison, 
73 Manocha, \& Rubia, 2004; Jensen \& Kenny, 2004). However, no control group design and a

74 small sample size brought to weaken the validity of these positive effects of yoga intervention on

75 ADHD children's behavior and cognition. By utilizing a larger sample with a symptom-match

76 compared group, the benefits of yoga exercise on alleviating the cognitive deficits of ADHD can

77 be further identified.

78 Physical activity intervention has been found to derive positive changes in behavioral

79 structures and cognitive function among children with ADHD, which are reflected in reduced

80 impulsivity, anxiety, and improved attention (Chang, Hung, Huang, Hatfield, \& Hung, 2014;

81 Huang et al., 2014; Smith et al., 2013; Verret, Guay, Berthiaume, Gardiner, \& Béliveau, 2012).

82 Additionally, for children with ADHD, even a single bout of exercise has been found to

83 contribute to response preparation (Chuang, Chang, Tsai, Huang, \& Hung, 2015) and task

84 switching (Hung, Huang, Tsai, Chang, \& Hung, 2016), and physical fitness has been associated

85 with baseline cortical activity (Huang, Huang, Hung, Shih, \& Hung, 2015) and inhibitory ability

86 (Tsai et al., in press). The mechanisms of physical activity effects on ADHD children's cognitive

87 function may be due to brain structure changes, enhanced neurotransmitters, and arousal

88 regulation (Lustig, Shah, Seidler, Reuter-Lorenz; 2009; Tang, Chu, Hui, Helmeste, \& Law,

89 2008). Like physical activity, yoga has been found to have beneficial impacts on neurological

90 and physiological activity and behavior in a range of populations. The reported benefits of yoga 
91 include increased slow-frequency brain wave activity (Arambula, Peper, Kawakami, \& Gibney,

92 2001); favorable profiles on heart rate (HR) variability, depression, perceived stress, and superior

93 aerobic fitness (Satin, Linden, \& Millman, 2014); and significant decrement of cortisol and

94 increment in brain-derived neurotropic factor (BDNF), serotonin, and dopamine (Pal, Singh,

95 Chatterjee, \& Saha, 2014). In essence, the practice of yoga exercise elicits reduced activation of

96 the sympathetic nervous system and increased activation of the parasympathetic nervous system

97 resulting in a sense of equilibrium into the body and mind, and increased emotional self-

98 regulation (Streeter, Gerbarg, Saper, Ciraulo, \& Brown, 2012). Given that abnormal attention

99 and over-impulsivity characteristics have been considered as major symptoms of ADHD, these

100 previous studies provide compelling empirical evidence for using yoga exercise in ADHD

101 treatment.

102 Although a few studies have reported potential associations between yoga exercise and

103 improved cognitive functions of children with ADHD (Jensen \& Kenny, 2004), these findings

104 are required to be replicated to warrant the use of yoga as an effective complementary treatment

105 for this population. Based on evidence revealing deviant patterns in behavioral impulsivity,

106 memory retrieval, sustain attention, stimuli differentiation, and decision making are relevant to

107 ADHD (Barkley, 1997; Bellgrove, Hawi, \& Robertson, 2006), the current study further

108 examined the effects of yoga exercise on the sustained attention and discrimination functions 
109 among ADHD children. Any changes in sustained attention and discrimination function observed

110 after yoga exercise could be used to identify the effects of yoga on improved ADHD symptoms.

111 Given the results of the studies briefly reviewed above, physical activity including yoga exercise

112 could contribute to reduced hyperactivity and inattention (Harrison et al., 2004; Jensen \& Kenny,

113 2004; Steiner et al., 2013), as well as increased interference control, attention shifting, and

114 consistency in response speed among individuals with ADHD (Halperin et al., 2014). Reduced

115 hyperactivity, increased attention and response consistency could contribute to better sustained

116 attention while increased interference control and attention shifting should enhance

117 discrimination function which involves inhibition and selective attention.

118 . This study hypothesized that yoga exercise could benefit the sustained attention and

119 discrimination function of children with ADHD by using the Visual Pursuit Test and the

120 Determination Test. The Visual Pursuit Test is usually used to assess visual perception involving

121 sustained attention and the Determination Test is used to evaluate the ability to determine

122 multiple-choice reaction requiring inhibitory ability and selective attention. Although the two

123 tests have not been utilized among individuals with ADHD, their availability has been reported

124 in clinical populations (Kober et al., 2013) and young athletes (Dogan, 2009). Such a study could

125 be critically important in laying the ground work for both scientific research and clinical

126 application. 


\section{Participants}

Fifty participants were recruited via flyers posted in relevant locations, referrals given to the

130

children's parents by their elementary schools, and a number of orientations conducted to

131 introduce the project. All participants were from schools located in suburban areas of a city

132 where most families were the middle to high socioeconomic populations. The inclusion criteria

133 were as follows: children aged between 8 and 12 years old who had been diagnosed with ADHD

134 by their own psychiatric physicians and had been confirmed by school pediatricians. All the

135 various subtypes of ADHD (inattentive, hyperactivity/impulsivity, combined) were included

136 regardless of whether he or she was receiving medication for ADHD symptoms. The exclusion

137 criteria were as follows: a) comorbid conditions such as conduct/oppositional defiant disorder,

138

autism spectrum disorders, or serious affective disorders, b) a personal history of brain injury or

139

neurological disorders, and c) currently taking sedatives or other mood altering medications

140

other than the stimulants typically prescribed for ADHD. All the children were assigned to one

141 of two groups according to their school districts: the yoga exercise group $(n=25)$ and the control

142 group $(n=25)$. One participant withdrew from the yoga exercise group due to personal

143 consideration. Figure 1 shows the flow diagram for the progress of all participants through the

144 study. Participants were instructed to refrain from medications and caffeine intake for at least 24 
145 hours prior to undergoing the various tests administered as part of the study. Written informed

146 consent was provided by the parents and the children following a full explanation of the study,

147 which was reviewed and approved by the Research Ethics Committee of National Taiwan

148 University prior to the experiment.

\section{Measures}

150

Visual Pursuit Test. The Visual Pursuit Test of the Vienna Test System (Schuhfried GmbH,

151 Austria), a computerized psychological assessment tool well established in psychological

152 diagnostics (Schmid, Sauter, Stepansky, Lobentanz, \& Zeitlhofer, 2005), was used in this study.

153 It is designed as a line tracking test and used for the registration of concentrated targeted

154 perception and selective attention in the visual area. Hence, the performance in this test requires

155 the ability of selective and sustained attention. It consisted of 54 different items; in each item, an

156 array of nine entwined dark lines leading to nine different endpoints was presented on a light

157 background computer screen. The starting point of one out of the nine lines was marked and the

158 participant was asked to follow this line with their eyes to find the corresponding endpoint as

159 quickly as possible by pressing one of nine number buttons on a response panel. The screen was

160 shown for four seconds and then disappeared. Therefore, items to which the participant did not

161 answer correctly or did not respond within the four seconds were reported as incorrect responses.

162 The performance of the participant was scored automatically, considering the number of correct 
163 answers and mean RT for correct answers. The test duration for each participant was

164 approximately 10 min per run.

165 Determination Test. The Determination Test has been used to assess the discrimination

166 ability for reaction speed, attention deficits, and reactive stress tolerance in the presence of

167 continuous but rapidly changing acoustic and optical stimuli (Shmygalev et al., 2011). In this

168 study, the participant's task was to react as quickly as possible to visual or acoustic stimuli by

169 pressing the corresponding buttons on the response panel. There were five visual stimuli colored

170 white, yellow, red, green and blue, which appeared in an upper and a lower row on the screen.

171 The reaction buttons assigned to these five colors were arranged on the response panel in such a

172 way that the participant could use both hands. There were two additional visual stimuli, in the

173 form of white, rectangular, visually distinct fields that appeared in the bottom left- and right-

174 hand corners of the screen, to which the participant had to react by pressing the corresponding

175 (left or right) foot pedal. Two acoustic stimuli (high and low tone) were assigned to the two

176 "sound" buttons in the middle of the panel. The lower, rectangular black button was pressed for a

177 low tone and the upper rectangular grey button for a high tone. The visual stimuli were presented

178 on the screen and the acoustic stimuli were presented via headphones. The duration of stimulus

179 presentation depended on the respondent's mean RT for the previous eight trials. If the response

180 to a stimulus was not correct, the RT was doubled for the purpose of calculating the duration of 
181 the next stimulus. This test contained 180 trials with 20 trials for each stimulus. The number of

182 correct trials was utilized to calculate the accuracy rate and RT of each correct response was

183 reported. The duration for the test was approximately $10 \mathrm{~min}$ for each participant.

184 Physical Fitness. The participants were instructed to not engage in any intense physical

185 activity or take any stimulant medication on the day before the evaluations. In addition to weight

186 and height, the physical fitness of each participant was estimated, including flexibility, muscular

187 endurance, power, and cardiovascular fitness. The fitness assessment includes measures of

188 flexibility (sit and reach test), muscular endurance (sit-ups in $1 \mathrm{~min}$ ), power (standing long jump),

189 and cardiovascular fitness (a half-mile run in the fastest possible time). The four subsets of

190 fitness assessment of all the participants were converted into standardized T-scores, and the

191 physical fitness score was computed as the mean of the scores on these fitness subsets.

192 Yoga Exercise Intervention

193 The manipulation of yoga activity followed the American Physical Therapy Association

194 guidelines for working with children (Galantino, Galbavy, \& Quinn, 2008). Each lesson for yoga

195 activity lasted for $40 \mathrm{~min}$, twice a week for eight weeks, was led by a nationally certified yoga

196 instructor, and was conducted in a dance studio with an average temperature of $24-26^{\circ} \mathrm{C}$. The

197 yoga activity session consisted of a 10-minute stretching and warming-up period followed by a

198 20-minute yoga activity, which included concentration and balance, improved attention, and 
199 breath and body awareness. Finally, each session ended with a 10-minute cooling-down period

200 including balancing, flexibility, and relaxation exercises. This clinical trial was approved by the

201 Chinese Ethics Committee of Registering Clinical Trials. During the entire session, each

202 participant's HR was recorded at one-min intervals by using a Polar HR monitor (Mode ZW 60

203 GT5; Cardiosprt, United Kingdom). The HR values during the periods of warming-up, main

204 activity, and cooling-down were calculated respectively. The intensity of the main activity was

205 set at 50-60 percent of maximal HR (HRmax) according to the previous study suggesting that

206 this intensity level of aerobic exercise is beneficial to baseline cognition function of children

207 with ADHD (Huang et al., 2014). The HRmax was estimated using a formula " 220 - age" and

208 then the target HR for each participant was calculated. To monitor the intensity of the main

209 activity, the researchers examined the HR data of each participant during the period of main

210 activity after the completion of each lesson. For all the lessons, the participants reached at least

21150 percent and did not exceed 60 percent of their HRmax during the main activity. The instructor

212 was recommended to maintain her prescribed activity and intensity.

213 To test for exercise intensity manipulation, one-way analysis of variance (ANOVA) was

214 conducted to compare the HR differences among the periods of warming-up, main activity, and

215 cooling-down. The results showed a significant increase in HR during the period of main activity

216 for yoga exercise $(105.21 \pm 4.24 \mathrm{bpm})$, compared to the periods of warming-up and cooling-down 
$217(78.38 \pm 3.23,84.87 \pm 3.18 \mathrm{bpm}), F_{(2,46)}=306.04, p<.001$, and partial $\eta^{2}=0.93$. During the main

218 activity for the yoga exercise, the intensity of exercise was approximately 53 percent of HRmax.

219 Briefly stated, the exercise intensity of yoga activity reached a moderate level.

220 Procedure

221 The participants were invited to come to the laboratory with their parents on two separate

222 days. The children who were undergoing medical treatment were asked to refrain from

223 medication for at least $24 \mathrm{~h}$ prior to the experiment. On the first visit, the participant's parent(s)

224 and the participant signed an informed consent form, provided a health history, and filled out a

225 demographics questionnaire. Each eligible participant then entered the pre-test stage, which

226 consisted of performing the Visual Pursuit Test and the Determination Test and a physical fitness

227 assessment. Prior to the physical fitness tests, the participants and their parents were verbally

228 inquired to assess their or their children's physical readiness for the tests. For those participants

229 who expressed they were not physically well-prepared to take the physical fitness tests, we

230 rearranged the tests for them on other visits as soon as possible. The order of the Visual Pursuit

231 Test and the Determination Test was counterbalanced. For the Visual Pursuit Test, the given

232 participant was asked to find the end of a specified line as rapidly as possible by pressing the

233 corresponding number buttons on the response panel. Before the formal test, eight trials were

234 provided for practicing. If over three trials for which the responses were in error, the participant 
235 was asked to repeat the practicing trials until they were familiar with the test. Then, the formal

236 trials were administered.

237 For the Determination Test, the participant was instructed to perform according to the

238 various color stimuli and acoustic signals presented by pressing the corresponding buttons on the

239 response panel. At the instruction phase, step-by-step instructions gave the participants the

240 necessary information regarding the test. The instructions started by explaining the colored

241 buttons on the response panel. The participants were then introduced to the visual stimuli and

242 sounds; samples of these could be seen and heard by pressing the corresponding buttons. The

243 instruction phase was followed by a practice phase. If more than three errors were made or if no

244 response was made within 45 seconds on three successive occasions, the practice phase was

245 automatically interrupted and the respondent was instructed to consult the test administrator. The

246 administrator could, if necessary, restart the instruction phase in order to ensure that the

247 instructions were fully understood. Then, the formal trials were conducted to the participants. As

248 these two tests were completed, the height and weight of the participants were measured and the

249 fitness assessment followed after warm-up exercises and detailed explanations of each testing

250 protocol. Participants were allowed to rest between each testing to avoid fatigue.

251 Participants in the yoga exercise group underwent an eight-week yoga exercise program that

252 consisted of two 40-min sessions per week as an after school program. In contrast to the exercise 
253 group, the participants in the control group were simply instructed to maintain their normal life

254 without participating in regular physical activity programs. Within one week of completing the

255 yoga exercise program, all the participants were invited to visit the laboratory for the second time.

256 Each participant was asked to perform the Visual Pursuit Test and the Determination Test again

257 for the comparison of pre- and post-test scores.

258 Statistical Analysis

259 To ensure that any potential confounds would be homogenous for both the exercise and

260 control groups, independent t-tests or chi-square tests were used to analyze for continuous or

261 discrete scales of demographic data, respectively, to compare between the two groups. Next, the

262 effects of yoga exercise on the performance in the Visual Pursuit Test and the Determination

263 Test were examined by 2 (Group: exercise, control) $\times 2$ (Time: pre-test, post-test) mixed design

264 ANOVAs. Following the ANOVAs, multiple comparisons with Bonferroni-Holm adjustments

265 were applied to control for experiment-associated inflation of type 1 error for small sample sizes.

266 Effect size (ES) values were calculated according to Cohen's d and partial eta-square $\left(\eta^{2}\right)$ for the

267 significant main effects and interactions. For all statistical analyses, a significance level of .05

268 was used prior to the adjustment.

270 Demographic Analyses 
271 The analyses results indicated that there were no significant differences between the groups

272 in terms of weight, height, body mass index (BMI), age, intelligence quotient (IQ),

273 cardiovascular fitness, muscular strength, muscular endurance, flexibility, and total physical

274 fitness, $t_{47}<1.07, p>.05$. In addition, in terms of gender, grade, type, and medicine intake for

275 both groups, $x^{2}<2.69, p>.05$, suggesting that the two groups were homogenous. Table 1

276 summarizes the demographic characteristics and physical fitness of the participants in both

277 groups.

278 The Visual Pursuit Test

279 As the accuracy rate of the Visual Pursuit Test presented in Table 2, a mixed design

280 ANOVA revealed no main effects of Group and Time. However, a significant interaction of

281 Group by Time was found, $F_{(1,47)}=4.26, p=.045$, partial $\eta^{2}=0.08$, exhibiting that the yoga

282 exercise group yielded a higher accuracy rate at the post-test than the control group, $t_{47}=2.70, p$

$283=.010, d=0.78$, along with no group differences observed at the pre-test. Furthermore, the

284 exercise group reported an increased accuracy rate after the yoga intervention, $t_{23}=-2.12, p=$

$2850.045, d=-0.69$, while no change in the accuracy rate was found for the control group, $t_{24}=0.86$,

$286 p=.397$.

287 For the RT data, the analysis reported a main effect of Group, $F_{(1,47)}=12.85, p=.001$,

288 partial $\eta^{2}=0.22$, revealing faster RT for the exercise group than the control group. Also, a main 
289 effect of Time was observed, $F_{(1,47)}=12.56, p=.001$, partial $\eta^{2}=0.21$, indicating faster $\mathrm{RT}$ at

290 the post-test than the pre-test. A significant interaction of Group by Time was found, $F_{(1,47)}=$

$2918.20, p=.006$, partial $\eta^{2}=0.15$, demonstrating that the yoga exercise group yielded a faster RT

292 at the post-test than the control group, $t_{47}=-4.18, p<.001, d=-1.20$, along with no group

293 differences observed at the pre-test. Additionally, the exercise group reported a decreased RT

294 after the yoga intervention, $t_{23}=4.12, p<.001, d=1.29$, while no $\mathrm{RT}$ change was found for the

295 control group, $t_{24}=0.54, p=.597$.

296 The Determination Test

297 In terms of the response accuracy of the Determination Test presented in Table 2, ANOVA

298 revealed a main effect of Time, $F_{(1,47)}=5.32, p=.026$, partial $\eta^{2}=0.10$, indicating a higher

299 accuracy rate at the post-test than the pre-test. A significant interaction of Group by Time was

300 reported, $F_{(1,47)}=17.48, p<.05$, partial $\eta^{2}=0.27$, revealing that the yoga exercise group yielded

301 a higher response accuracy at the post-test than the control group, $t_{47}=3.74, p<.001, d=1.09$,

302 along with no group differences observed at the pre-test. Furthermore, the exercise group

303 reported an increased response accuracy after the yoga intervention, $t_{23}=-5.78, p<.001, d=$ -

3041.22 , while no change in the response accuracy was found for the control group, $t_{24}=1.15, p=$

3050.263.

306 For the RT data, the analysis revealed a main effect of Group, $F_{(1,47)}=9.29, p=.004$, 
307 partial $\eta^{2}=0.17$, exhibiting faster RT for the exercise group than the control group. Also, a main

308 effect of Time was reported, $F_{(1,47)}=4.45, p=.040$, partial $\eta^{2}=0.09$, indicating faster RT at the

309 post-test than the pre-test. A significant interaction of Group by Time was observed, $F_{(1,47)}=$

$3104.79, p=.034$, and partial $\eta^{2}=0.09$, demonstrating that the yoga exercise group yielded a faster

311 RT at the post-test than the control group, $t_{47}=-4.26, p<.001, d=-1.25$, along with no group

312 differences observed at the pre-test. Furthermore, the exercise group reported a decreased RT

313 after the yoga intervention, $t_{23}=4.78, p<.001, d=1.26$, while no RT change was found for the

314 control group, $t_{24}=-0.05, p=.964$.

\section{Discussion}

The findings of this study are consistent with previous research (Cerrillo-Urbina et al.,

317 2015), revealing beneficial effects of yoga exercise on the core symptoms of children with

318 ADHD, such as sustained attention and discrimination ability. With HR monitoring applied to

ensure a moderate level of exercise intensity, the results showed that the yoga exercise program

320

exerted a positive impact on RT and response accuracy at the Visual Pursuit Test and the

321 Determination Test, whereas no such influences were found for the control group. The results

322 were promising because this study reported the homogeneity of demographic characteristics,

323 intelligence quotient, and physical fitness between the exercise and control groups at the baseline,

324 confirming that these variables might not confound our findings. Also, the participants were 
325 recruited from nearby residential areas where the average level of socioeconomic status was

326 similar. In particular, the present findings extend previous research by utilizing a larger sample,

327 symptom-match counterparts, and a moderate-intensity yoga exercise that involved aerobic,

328 flexibility, and perceptual-motor exercises.

329 In support of our hypothesis, the yoga exercise group demonstrated a faster RT and higher

330 response accuracy on the Visual Pursuit Test than the control group. The result that yoga

331 exercise enhanced selective and sustained attention in children with ADHD aligns with the

332 extant literature regarding effects of alternative therapies on ADHD symptoms (Majorek,

333 Tüchelmann, \& Heusser, 2004; Peck, Kehle, Bray, \& Theodore, 2005). These studies have

334 reported the facilitative impacts of yoga and massages on self-control, relaxation, and

335 concentration for children with ADHD. Yoga exercise typically conducts a variety of poses, deep

336 breath, concentration, and mental and physical relaxation which can positively regulate mental

337 states (Zipkin, 1985). It also tends to promote self-control, attention and concentration, self-

338 efficacy, body awareness, and stress reduction (Peck, et al., 2005). Recently, a meta-analytical

339 study has indicated that yoga exercise suggests substantial improvements such as alleviating

340 impulsivity, anxiety, and social problems and a mild improvement in attention and hyperactivity

341 for individuals with ADHD (Cerrillo-Urbina et al., 2015). The present findings are also

342 supported by other research regarding physical exercise effects on children with ADHD (Smith 
343 et al., 2013; Verret et al., 2012), reporting that sustained attention improves with long-term

344 physical exercise. Further, Palmer, Miller and Robinson (2013) revealed that after engaging in a

345 bout of movement program incorporating various motor skills, preschoolers exhibited better

346 ability to sustain attention compared to after being sedentary. Their finding highlights the

347 proposition that exercise requiring motor control is more likely to enhance sustained attention for

348 subsequent cognitive task because prefrontal brain regions involved in sustained attention are

349 activated after exercise (Budde et al., 2008). Yoga exercise always requires participants to

350 perform complex combinations of motor skills in a smooth and fluent manner, such as

351 controlling body posture and relative space using vestibular sense (Peck et al., 2005). Thus, the

352 yoga program in the current study, which involved posture control and motor skills, might

353 facilitate ADHD individuals' subsequent sustained attention performance of the Visual Pursuit

354 Test by activating the prefrontal cortex.

355 Moreover, the yoga exercise group exhibited a better performance on the Determination

356 Test than the control group, revealing that yoga exercise could be beneficial to children with

357 ADHD in terms of improving their discrimination ability. Given that the Determination Test

358 requires participants to quickly respond to different kinds of acoustic and optical stimuli, better

359 discrimination ability indicates that the participants exhibit greater inhibition toward the

360 interference of the previous stimulus, as well as faster and more accurate selection toward 
361 multiple stimuli and the corresponding reaction (Dogan, 2009). Previous studies have provided

362 supporting evidence regarding the positive effects of yoga exercise on the inhibitory function in

363 healthy children (Telles, Singh, Bhardwaj, Kumar, \& Balkrishna, 2013). Telles et al. (2013)

364 assessed the effects of yoga and physical exercise over three months and found that both

365 interventions improved performance in the Stroop task for healthy children. The cognitive

366 mechanisms involved in this task are attentional vitality and flexibility, as well as inhibition of a

367 dominant response. Therefore, similar to effects of physical exercise, yoga exercise has been

368 postulated to influence attention and inhibition, however, through different pathways. Previous

369 studies also support this notion, reporting that changes in bilateral putamen volumes of the dorsal

370 striatum and globus pallidus after a year long physical exercise were associated with inhibition

371 performance (Chaddock et al., 2012). On the other hand, the findings of neuroimaging studies

372 indicated that yoga practitioners exhibited increased blood flow to the dorsolateral prefrontal

373 cortex (Cohen et al., 2009). Hence the benefits of yoga practice in discrimination ability seem to

374 be associated with specific changes in particular brain areas.

375 Another possibility is that yoga exercise improves discrimination ability by improving

376 attention and information processing. Using a neuroelectrical approach, children with ADHD

377 that have greater motor ability exhibited better allocation of attentional resource and superior

378 efficiency of neuroprocessing than their counterparts with lower motor ability (Hung et al., 2013). 
379 After an eight-week aquatic exercise program, children with ADHD demonstrated an

380 improvement in accuracy for a widely used cognitive task that assesses behavioral inhibition

381 (Chang et al., 2014). The mechanisms underlying these benefits may involve complex neuro-

382 chemical changes and modified functioning of brain areas within the limbic circuit. For instance,

383 yoga exercise was found to be associated with decreased cortisol and increased BDNF, serotonin,

384 and dopamine (Pal et al., 2014). These biological mechanisms contribute to attention processing

385 and inhibition in terms of regulating arousal levels in fronto-striatocerebellar circuits and

386 enhancing the control of executive function (del Campo, Chamberlain, Sahakian, \& Robbins,

387 2011). In animal studies, increased BDNF levels in the hippocampus after exercise were found to

388 be related to elevated learning and memory processes (Vaynman, Ying, \& Gomez-Pinilla, 2004).

389 Although further studies are still required to confirm these findings, previous studies suggest that

390 yoga exercise may result in improved cognitive function in ADHD by the alteration of neuro-

391 chemical expressions.

392 Overall, the results of this study reported that yoga exercise consisting of breathing

393 manipulations, posture control and body balance, and concentration could enhance sustained

394 attention, interference control, and attention shifting among children with ADHD. These findings

395 suggest that physical activity combining various components of motor skills, body control, and

396 concentration practice is beneficial to specific components of executive function of children with 
397 ADHD. Some limitations of this study warrant caution and can direct future research. First,

398 although this study controlled the homogeneity of the two groups in terms of gender, age, ADHD

399 type, BMI, physical fitness, medication, and intelligence, the non-randomized controlled trial

400 design and unbalanced gender proportions (i.e., primarily boys) may limit the validity of the

401 findings. Accordingly, the precise causal effects of yoga exercise on cognitive function in

402 ADHD require future researchers to utilize a randomized controlled trial design. Secondly,

403 medication use as well as time spent in video games, internet, and television watching may

404 confound yoga exercise effects on cognitive function of children with ADHD; further

405 examination is required by ruling out the influences of these factors. Thirdly, based on previous

406 studies revealing a linkage between greater aerobic fitness and better executive function in

407 children (Telles et al., 2013), the present study shows little evidence provided to interpret the

408 mechanism of yoga effects on cognitive function of children with ADHD without physical

409 fitness assessment after the 8-week yoga program. Fourthly, although the possibility is low, this

410 study could not completely rule out the possibility that the observed effect was a result of less

411 attention paid to the control group. Finally, future research may investigate and expand upon the

412 present findings by reporting the level of physical activity outside the exercise intervention and

413 applying cognitive and behavioral perspectives to the assessment of ADHD symptoms.

\section{Conclusion}


415 In conclusion, our positive findings are in accordance with previous studies demonstrating

416 that yoga exercise can be utilized as an alternative treatment for children with ADHD to reduce

417 attention and inhibition problems. Furthermore, this study is one of only a few studies that have

418 investigated yoga exercise effects on cognitive function in ADHD. To date, stimulant

419 medications appear to be easy interventions to improve functioning in children with ADHD;

420 however, some documented side-effects prevent parents from implementing them. Thus, yoga

421 exercise shows promise as an effective and low-risk treatment for improving long-term cognitive

422 and functional outcomes of ADHD individuals. Given the beneficial effects of yoga exercise

423 participation on certain important ADHD-related cognitive function, the utilization of yoga

424 exercise as an extra-curriculum or a curriculum is recommended to schools and the parents of

425 children with ADHD.

426

427

428

429

430

\section{References}

Arambula, P., Peper, E., Kawakami, M., \& Gibney, K. H. (2001). The physiological correlates of 
434 Barbaresi, W. J., Katusic, S. K., Colligan, R. C., Pankratz, S. C., Weaver, A. L., Weber, K. J.,

435

436

437

438

439

440

441

442

443

444

445

446

447

448

449

450

451

Mrazek, D. A., \& Jacobsen, S. J. (2002). How common is attention-deficit/hyperactivity disorder? Incidence in a population-based birth cohort in Rochester, Minn. Archives of Pediatrics \& Adolescent Medicine, 156, 217-224. doi:10.1001/archpedi.156.3.217

Barkley, R. A. (1997). Behavioral inhibition, sustained attention, and executive functions: Constructing a unifying theory of ADHD. Psychological Bulletin, 121, 65-94. doi:10.1037/0033-2909.121.1.65

Barry, R. J., Clarke, A. R., \& Johnstone, S. J. (2003). A review of electrophysiology in attentiondeficit/hyperactivity disorder: I. Qualitative and quantitative electroencephalography. Clinical Neurophysiology, 114, 171-183. doi:10.1016/S1388-2457(02)00362-0

Bellgrove, M. A., Hawi, Z., \& Robertson, I. H. (2006). The cognitive genetics of attention deficit hyperactivity disorder (ADHD): Sustained attention as a candidate phenotype. Cortex, 42, 838-845.

Budde, H., Voelcker-Rehage, C., Pietrabyk-Kendziorra, S., Ribeiro, P., \& Tidow, G. (2008). Acute coordinative exercise improves attentional performance in adolescents. Neuroscience Letters, 441, 219-223. doi: 10.1016/j.neulet.2008.06.024

Cerrillo-Urbina, A. J., García-Hermoso, A., Sánchez-López, M., Pardo-Guijarro, M. J., Santos Gómez , J. L., \& Martínez-Vizcaíno, V. (2015). The effects of physical exercise in 
452

453

454

455

456

457

458

459

460

461

462

463

464

465

466

467

468

469 children with attention deficit hyperactivity disorder: A systematic review and metaanalysis of randomized control trials. Child: Care, Health and Development, 41, 779-788.

doi: 10.1111/cch.12255

Chaddock, L., Hillman, C. H., Pontifex, M. B., Johnson, C. R., Raine, L. B., \& Kramer, A. F. (2012). Childhood aerobic fitness predicts cognitive performance one year later. Journal of Sports Sciences, 30, 421-430. doi: 10.1080/02640414.2011.647706

Chang, Y. K., Hung, C. L., Huang, C. J., Hatfield, B. D., \& Hung, T. M. (2014). Effects of an aquatic exercise program on inhibitory control in children with ADHD: A preliminary study. Archives of Clinical Neuropsychology, 29, 217-223. doi:10.1093/arclin/acu003

Chuang, L. Y., Chang, Y. K., Tsai, Y. J., Huang, C. J., \& Hung, T. M. (2015). Effects of acute aerobic exercise on response preparation in a Go/No Go task in children with ADHD: An event-related potential study. Journal of Sport and Health Science, 4, 82-88. doi: 10.1016/j.jshs.2014.11.002

Cohen, D. L., Wintering, N., Tolles, V., Townsend, R. R., Farrar, J. T., Galantino, M. L., \& Newberg, A. B. (2009). Cerebral blood flow effects of yoga training: Preliminary evaluation of 4 cases. Journal of Alternative and Complementary Medicine, 15, 9-14. doi: 10.1089/acm.2008.0008.

del Campo, N., Chamberlain, S. R., Sahakian, B. J., \& Robbins, T.W. (2011). The roles of 
470 dopamine and noradrenaline in the pathophysiology and treatment of attention-

471 deficit/hyperactivity disorder. Biological Psychiatry, 69, e145-e157.

472 doi:10.1016/j.biopsych.2011.02.036.

473 Diamond, A., \& Lee, K. (2011). Interventions shown to aid executive function development in

474 children 4-12 years Old. Science, 19, 959-964. doi: 10.1126/science.1204529

475 Dogan, B. (2009). Multiple-choice reaction and visual perception in female and male elite

476 athletes. Journal of Sports Medicine and Physical Fitness, 49, 91-96.

477 Froehlich, T. E., Lanphear, B. P., Epstein, J. N., Barbaresi, W. J., Katusic, S. K., \& Kahn, B. S.

478

479

a national sample of US children. Archives of Pediatrics and Adolescent Medicine, 161,

480

857-864. doi: 10.1001/archpedi.161.9.857

481

Galantino, M. L., Galbavy, R., \& Quinn, L. (2008). Therapeutic effects of yoga for children: A

482 systematic review of the literature. Pediatric Physical Therapy, 20, 66-80. doi:

483 10.1097/PEP.0b013e31815f1208

484 Halperin, J. M., Berwid, O. G., \& O’Neill, S. (2014). Healthy body, healthy mind? The 485 effectiveness of physical activity to treat ADHD in children. Child and Adolescent Psychiatric Clinics of North America, 23, 899-936. doi: 10.1016/j.chc.2014.05.005

487 Harpin, V. (2005). The effect of ADHD on the life of an individual, their family, and community 
488

489

490

491

492

493

494

495

496

497

498

499

500

501

502

503

504

505

from preschool to adult life. Archives of Disease in Childhood, 90(Suppl 1), i2-i7. doi:

10.1136/adc. 2004.059006

Harrison. L. J., Manocha, R., \& Rubia, K. (2004). Sahaja yoga meditation as a family treatment for children with attention deficit-hyperactivity disorder. Clinical Child Psychology and Psychiatry, 9, 479-497. doi: 10.1177/1359104504046155

Huang, C. W., Huang, C. J., Hung, C. L., Shih, C. H., \& Hung, T. M. (2015). Physical fitness and resting EEG in children with attention deficit hyperactivity disorder: An exploratory study. Journal of Psychophysiology, 29, 26-32.doi: 10.1027/0269-8803/a000131

Huang, C. J., Huang, C. W., Tsai, Y. J., Tsai, C. L., Chang, Y. K., \& Hung, T. M. (2014). A preliminary examination of aerobic exercise effects on resting EEG in children with ADHD. Journal of Attention Disorders, 1-6. doi: 10.1177/1087054714554611

Hung, C. L., Chang, Y. K., Chan, Y. S., Shih, C., Huang, C., \& Hung, T. (2013). Motor ability and inhibitory processes in children with ADHD: A neuroelectric study. Journal of Sport \& Exercise Psychology, 35, 322-328.

Hung, C. L., Huang, C. J., Tsai, Y. J., Chang, Y. K., \& Hung, T. M. (2016). Neuroelectric and behavioral effects of acute exercise on task switching in children with attentiondeficit/hyperactivity disorder. Frontiers in Psychology, 7:1589. doi:

10.3389/fpsyg.2016.01589 
506 Jensen, P. S., \& Kenny, D. T. (2004). The effects of yoga on the attention and behavior of boys with attention-deficit/hyperactivity disorder (ADHD). Journal of Attention Disorders, 7,

508 205-216. doi: $10.1177 / 108705470400700403$

509

510

511

512

513

514

515

516

517

518

519

520

521

522

523 term memory. The Online of Sport Psychology, 9, 34-42.

Kober, S. E., Wood, G., Hofer, D., Kreuzig, W., Kiefer, M., \& Neuper, C. (2013). Virtual reality in neurologic rehabilitation of spatial disorientation. Journal of NeuroEngineering and Rehabilitation, 10:17. doi: 10.1186/1743-0003-10-17

Lustig, C., Shah, P., Seidler, R., \& Reuter-Lorenz, P. A. (2009). Aging, training, and the brain: A review and future directions. Neuropsychology Review, 19, 504-522. doi: 10.1007/s11065-009-9119-9

Majorek, M., Tüchelmann, T., \& Heusser, P. (2004). Therapeutic eurythmy-movement therapy for children with attention deficit hyperactivity disorder (ADHD): A pilot study. Complementary Therapies in Nursing \& Midwifery, 10, 46-53. doi: 10.1016/S13536117(03)00087-8.

Pal, R., Singh, S. N., Chatterjee, A., \& Saha, M. (2014). Age-related changes in cardiovascular system, autonomic functions, and levels of BDNF of healthy active males: Role of yogic practice. Age, 36(4):9683. doi: 10.1007/s11357-014-9683-7 
524 Palmer, K. K., Miller, M. W., \& Robinson, L. E. (2013). Acute exercise enhances preschoolers'

525 ability to sustain attention. Journal of Sport \& Exercise Psychology, 35, 433-437.

526 Peck, H. L., Kehle, T., Bray, M. A., \& Theodore, L. A. (2005). Yoga as an intervention for

527 children with attention problems. School Psychology Review, 34, 415-424.

528 Pliszka, S. (2007). Practice parameter for the assessment and treatment of children and

529 adolescents with attention-deficit/hyperactivity disorder. Journal of the American

$530 \quad$ Academy of Child \& Adolescent Psychiatry, 46, 894-921. doi:

531 10.1097/chi.0b013e318054e724

532 Satin, J. R., Linden, W., \& Millman, R. D. (2014). Yoga and psychophysiological determinants

534

535 of cardiovascular health: Comparing yoga practitioners, runners, and sedentary individuals. Annals of Behavioral Medicine, 47, 231-241. doi: 10.1007/s12160-013-9542-

536 Schmid, G., Sauter, C., Stepansky, R., Lobentanz, I. S., \& Zeitlhofer, J. (2005). No influence on selected parameters of human visual perception of $1970 \mathrm{MHz}$ UMTS-like exposure. Bioelectromagnetics, 26, 243-250. doi: 10.1002/bem.20076 psychomotor and cognitive function. Drug and Alcohol Dependence, 117, 190-197. doi: 
543 Smith, A. L., Hoza, B., Linnea, K., McQuade, J. D., Tomb, M., Vaughn, A. J., Shoulberg, E. K.,

544

545

546

547

548

549

550

551

552

553

554

555

556

557

558

\& Hook, H. (2013). Pilot physical aactivity intervention reduces severity of ADHD symptoms in young children. Journal of Attention Disorders, 17, 70-82. doi: $10.1177 / 1087054711417395$

Steiner, N. J., Sidhu, T. K., Pop, P. G., Frenette, E. C., \& Perrin, E. C. (2013). Yoga in an urban school for children with emotional and behavioral disorders: A feasibility study. Journal of Child \& Family Studies, 22, 815-826. doi: 10.1007/s10826-012-9636-7

Streeter, C. C., Gerbarg, P. L., Saper, R. B., Ciraulo, D. A., \& Brown, R. P. (2012). Effects of yoga on the autonomic nervous system, gamma-aminobutyric-acid, and allostasis in epilepsy, depression, and post-traumatic stress disorder. Medical Hypotheses, 78, 571-579. doi: 10.1016/j.mehy.2012.01.021

Tang, S. W., Chu, E., Hui, T., Helmeste, D., \& Law, D. (2008). Influence of exercise on serum brain-derived neurotrophic factor concentrations in healthy human subjects. Neuroscience Letters, 431, 62-65. doi: 10.1016/j.neulet.2007.11.019.

Telles, S., Singh, N., Bhardwaj, A. K., Kumar, A., \& Balkrishna, A. (2013). Effect of yoga or physical exercise on physical, cognitive and emotional measures in children: A 

1030-1034. doi: 10.1111/j.1460-9568.2004.03720.x

568 Verret, C., Guay, M., Berthiaume, C., Gardiner, P., \& Béliveau, L. (2012). A physical activity program improves behavior and cognitive functions in children with ADHD: An exploratory study. Journal of Attention Disorders, 16, 71-80. doi: 
578

579

$580 \quad$ Table 1

581 Demographic characteristics of the participants

\begin{tabular}{|c|c|c|c|}
\hline Variable & $\begin{array}{l}\text { Exercise group } \\
(n=24 ; M[S D])\end{array}$ & $\begin{array}{l}\text { Control group } \\
(n=25 ; M[S D])\end{array}$ & $\begin{array}{c}\text { Total } \\
(n=49 ; M[S D])\end{array}$ \\
\hline Gender (male: female) & $19: 5$ & $19: 6$ & $38: 11$ \\
\hline Age (years) & $10.71[1.00]$ & $10.30[1.07]$ & $10.50[1.05]$ \\
\hline Height (cm) & $143.71[9.42]$ & $143.08[9.43]$ & $143.39[9.33]$ \\
\hline Weight (kg) & $39.88[7.48]$ & $40.00[6.96]$ & $39.94[7.14]$ \\
\hline BMI $\left(\mathrm{kg} / \mathrm{m}^{2}\right)$ & $19.15[1.97]$ & $19.50[2.81]$ & $19.33[2.41]$ \\
\hline IQ (score) & $100.79[6.96]$ & $102.32[9.98]$ & $101.57[8.58]$ \\
\hline Physical fitness ( $T$ score) & $49.70[.50]$ & $50.48[3.67]$ & $50.10[3.57]$ \\
\hline Cardiovascular fitness (second) & $282.90[28.09]$ & $289.70[30.64]$ & $286.37[29.31]$ \\
\hline Muscular strength $(\mathrm{cm})$ & $128.79[8.58]$ & $126.96[9.91]$ & $128.35[9.29]$ \\
\hline Muscular endurance (times) & $22.79[3.41]$ & $23.12[3.44]$ & $22.96[3.39]$ \\
\hline Flexibility $(\mathrm{cm})$ & $25.45[3.87]$ & $25.76[3.89]$ & $25.61[3.84]$ \\
\hline \multicolumn{4}{|l|}{ Grade $(n)$} \\
\hline Third & 1 & 4 & 5 \\
\hline Fourth & 11 & 10 & 21 \\
\hline Fifth & 7 & 7 & 14 \\
\hline Sixth & 5 & 4 & 9 \\
\hline \multicolumn{4}{|l|}{ ADHD type $(n)$} \\
\hline ADHD-I & 12 & 8 & 20 \\
\hline ADHD-HI & 7 & 13 & 20 \\
\hline ADHD-C & 5 & 4 & 9 \\
\hline Medication $(n)$ & 10 & 12 & 22 \\
\hline
\end{tabular}

Note. BMI: body mass index. The IQ (intelligence quotient) score was assessed by the

583

Wechsler Intelligence Scale for Children-IV. ADHD-I: predominantly inattentive subtype;

584

ADHD-HI: predominantly hyperactive-impulsive subtype; ADHD-C: combined

hyperactive-impulsive and inattentive subtype. 
586

587

588

589

590

591 Table 2

592 Means (SD) for the Visual Pursuit Test and the Determination Test

\begin{tabular}{cccccc}
\hline \multirow{2}{*}{ Variable } & \multicolumn{2}{c}{ Exercise group } & & \multicolumn{2}{c}{ Control group } \\
\cline { 2 - 4 } & Pre-test & Post-test & Pre-test & Post-test \\
\hline Visual Pursuit Test & & & & \\
Accuracy rate (\%) & $95.60(5.43)$ & $98.61(2.95)$ & $96.45(3.89)$ & $95.12(5.63)$ \\
Reaction time (sec.) & $1.31(0.11)$ & $1.16(0.12)$ & $1.34(0.11)$ & $1.32(0.15)$ \\
Determination Test & & & & \\
Accuracy rate (\%) & $72.26(10.63)$ & $85.48(11.43)$ & $75.72(15.18)$ & $71.90(13.80)$ \\
Reaction time (sec.) & $0.95(0.07)$ & $0.86(0.07)$ & $0.99(0.17)$ & $0.99(0.13)$ \\
\hline
\end{tabular}

593

594

595

596

597

598

599

600

601

602

603

604

605

606

607

608 
609

610

611

612

613

614

615

616

617

618

619

620

\section{Enrollment}

Assessed for eligibility $(n=50)$

621

622

623

624

625

626

627

628

629

630

631

632

633

634

635

636

637

638

639

640

641

642

643

644
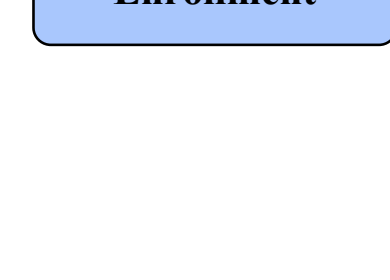

Non-randomized $(\mathrm{n}=50)$

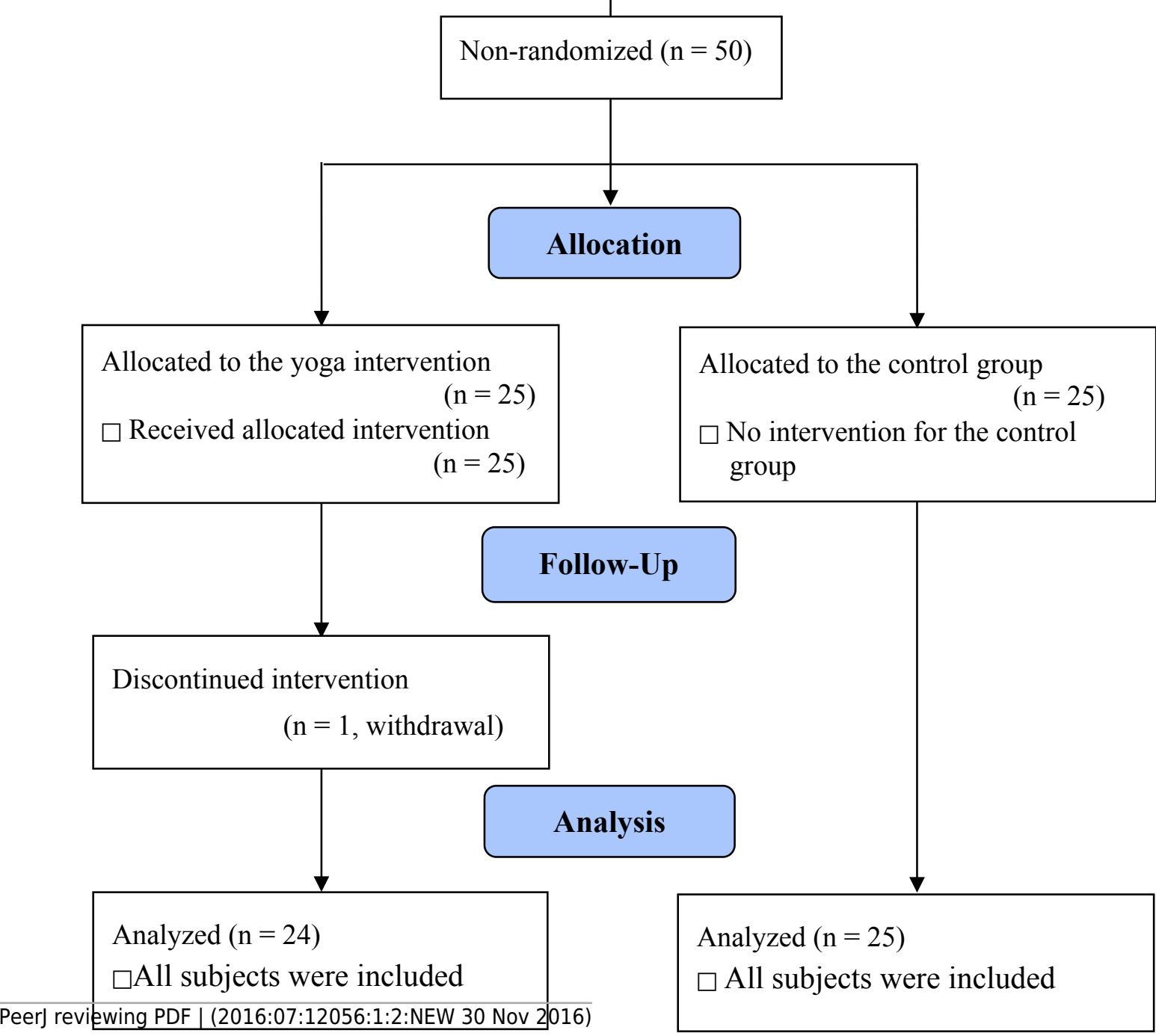

Allocated to the yoga intervention

$(n=25)$

$\square$ Received allocated intervention

$(n=25)$

Excluded $(\mathrm{n}=0)$

Not meeting inclusion criteria $(\mathrm{n}=0)$

Declined to participate $(n=0)$ 
645

646

647

648

649

650

Figure 1. The flow diagram for the progress of all participants through the trial.

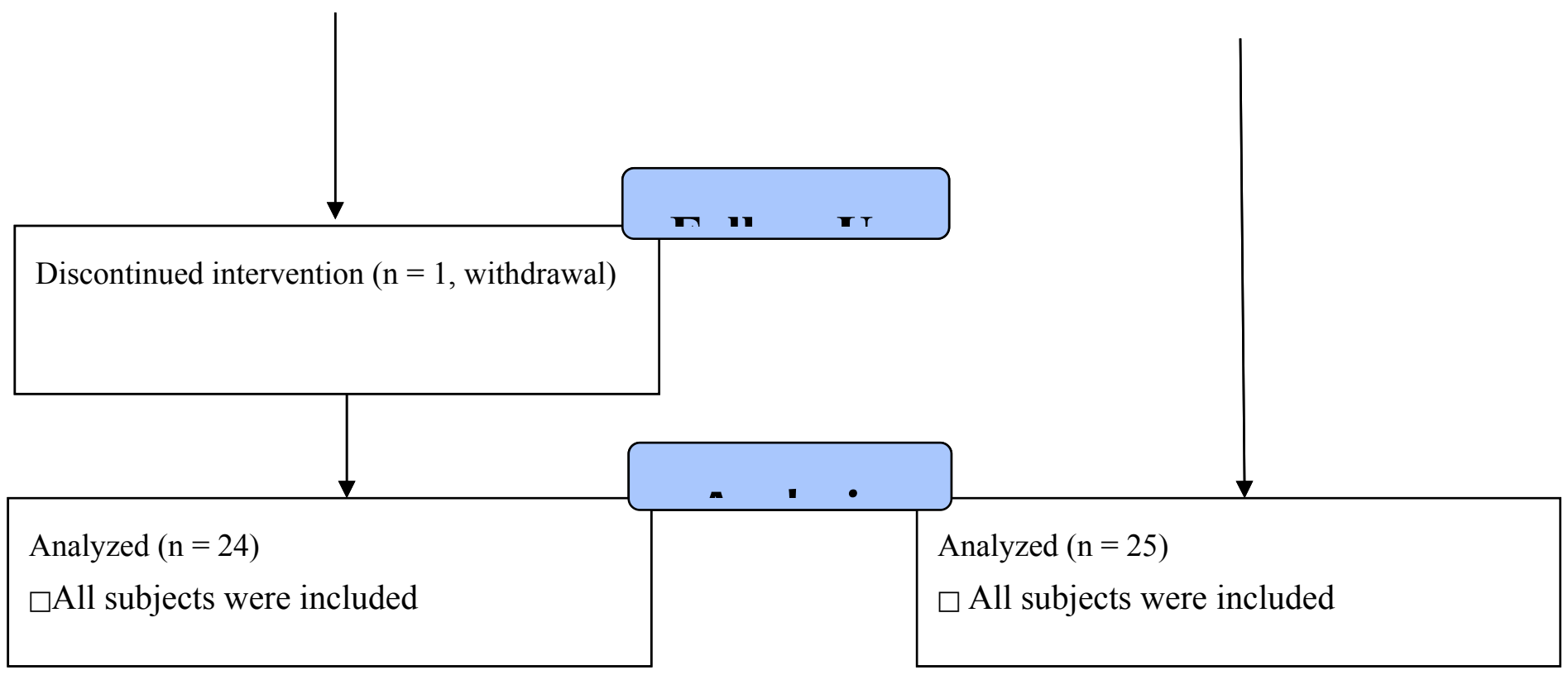

